\title{
Effectors, Effectors et encore des Effectors: The XIV International Congress on Molecular-Plant Microbe Interactions, Quebec
}

\author{
Jonathan D. Walton, ${ }^{1}$ Tyler J. Avis, ${ }^{2}$ James R. Alfano, ${ }^{3}$ Mark Gijzen, ${ }^{4}$ Pietro Spanu, ${ }^{5}$ Kim Hammond- \\ Kosack, ${ }^{6}$ and Federico Sánchez ${ }^{7}$
}

${ }^{1}$ Department of Energy-Plant Research Laboratory, Michigan State University, E. Lansing, MI, U.S.A.; ${ }^{2}$ Department of Chemistry, Carleton University, Ottawa, Canada; ${ }^{3}$ Center for Plant Science Innovation, University of Nebraska, Lincoln, NE, U.S.A.; ${ }^{4}$ Agriculture and Agri-Food Canada, London, Canada; ${ }^{5}$ Department of Life Sciences, Imperial College, London, U.K.; ${ }^{6}$ Department of Plant Pathology and Microbiology, Rothamsted Research, Harpenden, U.K.; ${ }^{7}$ Departamento de Biología Molecular de Plantas, Instituto de Biotecnología, Universidad Nacional Autónoma de México, Cuernavaca, Mexico

The IS-MPMI held its fourteenth meeting in Quebec City July 19-23, 2009. There were 956 attendees from 49 countries. Just over $50 \%$ of the participants were students or postdoctoral associates. Hani Antoun (Université Laval, Canada) served as Program Chair, capably assisted by the Local Organizing Committee and the IS-MPMI International Advisory Board. There were eight plenary sessions, 18 concurrent sessions, and over 700 posters.

Luis Sequeira, professor emeritus of plant pathology at the University of Wisconsin-Madison, a founder and former president of IS-MPMI and a former editor of this journal, gave a stirring "call to action" opening address the first night. Dr. Sequeira expressed his satisfaction with the growth and quality of the scientific research presented over the years at the ISMPMI Congresses and in the journal but reminded attendees of the need to enhance the impact and societal relevance of ISMPMI by "translating" that research into benefits for humankind. His call for translational research became one of the recurring themes of the meeting, as other speakers responded to Dr. Sequeira's comments in their own presentations over the next four days. The importance of charitable organizations that foster translational research for agricultural development and food security, such as the Two Blades Foundation and the Bill and Melinda Gates Foundation, were mentioned by others as emerging examples of how this type of work could be supported outside of the mainstream government grant-based funding model.

One example of successful translational research was given at the plenary session on "Technology Transfer" by Dennis Gonsalves (USDA, Hilo, Hawaii), who recounted the history of the successful introduction of transgenic virus-resistant papaya in Hawaii.

Also on the opening night, Jeff Dangl (University of North Carolina, U.S.A.), winner of this year's MPMI Award, posed several questions for current and future work on plant diseases. What is the natural diversity of virulence effectors and factors? What is the mechanism by which NB-LRR proteins function? How do we define active nucleotide binding-leucine-rich repeat (NB-LRR) alleles? Examples of answers to these kinds of questions were given from Dangl's recent research on RARl and the role of HSP90 in plant disease resistance.
The age of effectors.

Even in a specialized society like IS-MPMI, the flavor and scientific emphasis is constantly changing. Exactly where the spotlight falls and where the greatest momentum of research is varies from meeting to meeting. The major theme at this conference was effector proteins-structure, function, transport, location-from all classes of pathogens.

Pierre De Wit (Wageningen University, Netherlands) opened the scientific sessions with a demonstration that orthologous effector proteins from Cladosporium fulvum (a pathogen of tomato) and Mycosphaerella fijiensis (a pathogen of banana) share common recognition properties. Thus, the $M$. fijiensis ortholog to $C$. fulvum Avr4 triggers a hypersensitive response on Cf-4 tomato plants. Likewise, the M. fijiensis ortholog to $C$. fulvum Ecp2 triggers a hypersensitive response on Cf-Ecp2 tomato plants. These findings appear to blur the line between pathogen-associated molecular pattern (PAMP)-triggered immunity (PTI) and effector-triggered immunity (ETI) and offer new prospects for disease control by interspecies transfer of resistance $(R)$ genes.

Oomycete effectors have recently captured much attention due to the discovery of conserved host targeting signals that are characteristic of rapidly evolving effector gene families in species of genus Phytophthora. So far, we know of at least twelve Avr factors from three different oomycete species ( $P$. sojae, $P$. infestans, and Hyaloperonospora arabidopsidis) that carry the RxLR host-targeting motif located just downstream of the signal peptide. Presentations by Sophien Kamoun (Sainsbury Laboratory, U.K.), Brett Tyler (Virginia Bioinformatics Institute, U.S.A.), and Paul Birch (Scottish Crop Research Institute, Dundee) provided the latest details on RxLR effectors, such as their clustering in particular genome regions, how they may enter host cells, and what some of their targets may be.

The $P$. infestans genome was described by Dr. Kamoun as an exaggeration of features that were evident in the genomes of $P$. sojae and $P$. ramorum, which were the first two oomycete genomes completed some five years ago. Repeat-driven expansion of intergenic regions is especially evident in areas in which conserved synteny has broken down among the three species. These extensive repeat-rich regions are populated by genes encoding RxLR effectors and are distinguished from the 
"core genome," which is defined by some $40 \%$ of all $P$. infestans genes that have clear orthologs in the other two species. Thus, oomycete genomes can be broadly divided into regions of high gene density and low repeat content with conserved synteny (the core genome), and repeat-rich regions of low gene density and high effector content (the "plastic genome"). Similar clustering of effector genes in fungal plant pathogens, such as the smut fungi Ustilago maydis, U. scitaminea, and Sporisorium reilianum, was noted by Regine Kahmann (Max Planck Institute, Marburg, Germany). She described her recent results on the complex clustered (and redundant) effectors of $U$. maydis. More than 170 genes encode predicted secreted and infection-upregulated effectors in $U$. maydis. Effector mutants are blocked at different stages of infection. Yeast two-hybrid screens to identify host interactors have remained technically challenging; this might be because individual effectors have partially overlapping functions and influence each other's phenotypes.

More similarities between oomycete and fungal effectors were suggested by experiments that probed their mechanism of entry of into host cells. Dr. Tyler noted that the RxLR motif of $P$. sojae Avr1b can be functionally interchanged with sequence segments from fungal effectors, and he suggested a common mode of entry via endocytosis and endosome formation. Data was presented that showed specific binding of oomycete and fungal effectors to particular phosphatidyl inositol phosphates. These lipids are components of the host plasma membrane and have roles in signaling and defense. Dr. Tyler showed that fungal and oomycete avr proteins bind phosphatidyl inositolphosphates, which facilitates their uptake into host cells.

So-called hemibiotrophic and necrotrophic pathogens have an arsenal of effectors as well. Leptosphaeria maculans, a fungal necrotroph and endophyte of Brassica species, has numerous putative effector genes that are concentrated within A-T rich isochores in the genome (Thierry Rouxel, INRA, Versailles, France). The L. maculans effector genes, which are upregulated during infection, are related to the SIX (secreted in xylem) effector proteins of Fusarium oxysporum, which Frank Takken (University of Amsterdam, Netherlands) discussed as an example of "iterative evolution" of effectors and their targets in the arms race between $F$. oxysporum and tomato. Jeff Ellis and Peter Dodds (CSIRO, Canberra, Australia) brought attendees up to date on their research on the effectors and $R$ genes of the classic biotrophic flax rust system, in which the effector proteins and $R$ gene products interact directly inside the host cell. The motif or motifs controlling uptake of the rust effectors have been narrowed down to a small region near the $\mathrm{N}$ terminus. Development of a rust transformation strategy by the same group should greatly facilitate further progress.

Gitta Coaker (University of California, Davis, U.S.A.) and David Mackey (Ohio State University, U.S.A.) both gave talks on the Arabidopsis RIN4 protein. RIN4 interacts with multiple $\mathrm{R}$ proteins, is targeted by multiple type III effectors, and acts as a negative regulator of PTI. It has been a bit of a mystery why effectors would target RIN4 if disabling it triggers PTI. These talks provided evidence that RIN4 may play additional roles in innate immunity. Dr. Coaker's talk summarized her recent identification of six proteins in the RIN4 protein complex. Two of these proteins are the plasma membrane $\mathrm{H}^{+}$-ATPases AHA1 and AHA2. They also found that rin4 mutant plants cannot reopen their stomata when treated with Pseudomonas syringae. Taken together, these data suggest that RIN4 and an ATPase pump function in concert to regulate stomata during innate immunity. Mackey's talk described the identification of functional domains within RIN4. Interestingly, several truncated portions of RIN4 suppress PTI, suggesting that modification of RIN4 by type III effectors may suppress PTI.
P. syringae effector AvrPphB was the topic of a talk by JianMin Zhou (National Institute of Biological Sciences, Beijing, China). AvrPphB is a cysteine protease that cleaves the Arabidopsis PBS1 kinase. Dr. Zhou showed that it also cleaves other protein kinases, including BIK1, earlier shown to be involved in resistance to the fungus Botrytis cinerea. Dr. Zhou and colleagues determined that AvrPphB can suppress PTI. Interestingly, BIK1 is phosphorylated upon activation of FLS2. It appears that the relationship between PBS1 and BIK1 (and the other kinases) is analogous to the relationship between the Pto kinase and the PAMP receptor kinases, which are all targeted by the type III effector AvrPto. That is, AvrPto inhibits the Pto kinase-inducing Prf-dependent ETI, but its virulence targets appear to be the PAMP receptor kinases, while AvrPphB cleaves PBS1 to induce RPS5-dependent ETI.

The talk by Brad Day (Michigan State University, U.S.A.) emphasized the importance of actin dynamics in the response of plants to both bacteria and fungi. Actin depolymerizing factor AtADF4 was identified as being specifically required for resistance triggered by the $P$. syringae effector AvrPphB but not AvrRpt 2 or AvrB.

\section{The business of AvrBs3.}

New research on the bacterial effectors of Xanthomonas spp. caused a stir, especially results presented by Thomas Lahaye and Ulla Bonas (Martin-Luther-University, Halle, Germany) and independently by Adam Bogdanove and Matthew Moscou and colleagues at Iowa State University, U.S.A., on AvrBs3 from Xanthomonas campestris pv. vesicatoria. Although AvrBs3 was identified some 20 years ago, exactly how this effector works has been a longstanding puzzle. Among the features of the AvrBs3 sequence is an embedded repetitive region that binds to host gene promoters to activate their transcription. Targets that are upregulated by AvrBs3 (called upa genes) have a "upa-box" in their promoters to which the AvrBs3 effector protein binds. The DNA binding domain of AvrBs3 contains 17.5 copies of a near-perfect repeat of 34 amino acids. Within the repeated segments, a pair of amino acids in positions 12 and 13 defines the specificity of binding. By analysis of the frequency of binding of discrete amino acid pairs to each of the four DNA bases, a direct link between amino acid sequence and DNA binding activity was established. For example, a Cys and an Asp in positions 12 and 13 correspond to the nucleotide cytosine in the cognate position in the upa box. This unusual and straightforward correspondence between amino acid and nucleotide sequence raises many new possibilities for bioinformatic analyses and molecular engineering. For example, Dr. Lahaye suggested that multiple upa boxes could be arranged in tandem upstream of an $R$ gene such that the $R$ gene is induced by multiple AvrBs 3 members, possibly providing more durable resistance to xanthomonad pathogens. Brian Staskawicz (University of California, Berkeley, U.S.A.) also considered durable resistance to xanthomonads. In this case, conserved effectors can be identified in field isolates of $X$. vesicatoria, as are $R$ gene proteins that recognize these effectors to induce ETI. This would allow for the pyramiding of $R$ genes in plants that recognize the most conserved effectors in field isolates.

\section{Systemic responses.}

Although no session at the Congress was specifically devoted to the topic of systemic activation of defense responses (SAR), a wealth of new information was revealed during the meeting. Key players of the past, namely, salicylic acid (SA), methyl-SA, the NPR1 protein, and, more tentatively, DIR1 (Robin Cameron, McMaster University, Hamilton, Canada), were now joined by a new cast of protein players and diverse 
signaling molecules. Corina Vlot and Jane Parker (Max Planck Institute, Köln, Germany) revealed that EDS1, the lipase-like protein required for TIR-NBS-LRR (toll interleukin 1 receptornucleotide binding site-leucine-rich repeat) $\mathrm{R}$ protein function in Arabidopsis, is also required for SAR signal generation or early transmission or both, as well as for systemic signal recognition or propagation. In petiole exudates, this group identified seven secreted "AED" proteins whose presence was EDS1-dependent. In a study by Hak Jin and co-workers (Korea University, Seoul), local treatment of Arabidopsis leaves with the GDSL lipase 1 protein (GLIP1) was sufficient for the activation of systemic resistance. This function was independent of SA but required ethylene. In the phloem exudate of the GLIP1-treated plants, two lipid molecules (FA and Xs) were identified as the potential mobile signals. Local treatment with the FA-Xs elicited systemic resistance in an NPR1-dependent manner.

Jyoti Shah (University of North Texas, Denton, U.S.A.) used Arabidopsis petiole exudates collected after initial infection by avirulent $P$. syringae pv. tomato DC 3000 avrRpt 2 bacteria as a screening tool to identify novel compounds that act as longdistance SAR signals. The group identified the terpenoid dehydroabietinal from petiole exudates of pathogen-infected leaves, 10 pmol of which was sufficient to induce SAR. Induction was dependent on DIRI, SA, and NPRI.

Ho Won Jung and Jean Greenberg (University of Chicago, U.S.A.) discussed their latest results on azelaic acid, which they had earlier reported to be involved in systemic "priming." Azelaic acid acts downstream of SFD1 and FAD7, whereas a dirl mutant was insensitive to azelaic acid.

Finally, Murray Grant (University of Exeter, U.K.) presented a systems biology approach to elucidating time-dependent responses in systemically-induced leaves of Arabidopsis. Local photon generation associated with lipid peroxidation (approximately $2 \mathrm{~h}$ ) was rapidly followed by systemic jasmonateinduced gene responses $(<4 \mathrm{~h})$, and induction of genes associated with tryptophan-derived indole metabolism, in particular components of the glucosinolate and auxin biosynthetic pathways $(>7 \mathrm{~h})$. Although genetic disruption of indole biosynthesis or auxin polar transport compromised SAR and modified the systemic metabolome, it remains unclear which of these systemic responses actually contributes to SAR expression.

\section{PAMPs (MAMPs) and PTI.}

The field of plant-pathogen interactions now recognizes a fundamental distinction between PTI and ETI, and the similarities and differences between PTI and ETI was another recurring theme at the Congress. Well-studied PAMPs (which are also called MAMPs for microbe-associated molecular patterns) include bacterial lipopolysaccharide (LPS), bacterial flagellin (flg22), chitin, and bacterial elongation factor (EF$\mathrm{Tu})$. MAMPs are recognized in plants by pattern recognition receptors (PRR). Paul Schulze-Lefert (Max Planck Institute, Köln, Germany) presented evidence that a $\beta$-glucan receptor, GSRK, is another PRR involved in oomycete and fungal resistance. Yoshitake Desaki and colleagues (Meiji University, Japan) presented a poster on the role of LPS in "priming" rice defense responses. Cyril Zipfel (Sainsbury Laboratory, U.K.) used forward genetics to identify numerous genes that modulate the response of Arabidopsis to EF-Tu, a well-known PAMP whose receptor is the LRR receptor kinase EFR. Analysis of the genes indicates an unexpected role for the endoplasmic reticulum quality control machinery in PTI.

Sheng Yang He (Michigan State University, U.S.A.) updated the Congress on his work on coronatine-induced stomatal closure. Arabidopsis mutants lacking the FLS2 receptor for bacterial flagellin rescue the virulence defect of coronatine-deficient bacteria, suggesting that bacterial flagellin is the PAMP that triggers stomatal closure.

\section{Small RNAs.}

Much of our knowledge of RNA silencing comes from work on plant-virus interactions, including virus-induced gene silencing (VIGS). RNA interference and VIGS are now widely used for basic studies in plant biology, and at least 10 oral presentations and posters at the Congress reported the use of VIGS to address questions about the microbial interactions of various plant species, including legumes (Brett Ferguson, University of Queensland, Australia), rice (Xinshun Ding, Samuel Roberts Noble Foundation, Ardmore, OK, U.S.A.), and pepper (Jeena Hwang, Seoul National University, Korea). RNA silencing was the focus of both plenary and concurrent sessions. Olivier Voinnet (CNRS-Université de Strasbourg, France) spoke about small RNA pathways and how small RNAs mediate virus resistance. Arabidopsis has 10 Argonaute (AGO) paralogs, and AGO and RNA complexes accumulate in secreted vesicles called exosomes that derive from multivesicular bodies.

There is now substantial evidence that small plant RNAs have various endogenous roles, including mediation of responses to pathogens other than viruses. For example, microRNAs are emerging as key regulators of the symbiotic interaction between Medicago truncatula and Sinorhizobium meliloti (Martin Crespi, CNRS, Gif-sur-Yvette, France).

\section{Biocontrol and biosurfactants.}

Biocontrol and the complex ecological interactions between pathogens, symbionts, and plants continued to be a major theme at IS-MPMI congresses. This year, biosurfactants were a major topic. Microbial biosurfactants (e.g., rhamnolipids, cellobiose lipids, and lipopeptides) have been studied in various fields for their detergent and decontamination properties. The potential role of biosurfactants in biocontrol was addressed by Monica Höfte (Ghent University, Belgium); a cyclic lipopeptide (CLP) biosurfactant from a Pseudomonas strain was needed for its antagonistic activity. CLP was not only directly active against the pathogenic fungus Rhizoctonia solani, but it also seems necessary for the activity of the coproduced phenazine antibiotics. This may indicate that the biosurfactant helps with the bioavailability of the antibiotics, probably through interacting with (i.e., weakening) the fungal membrane. Along the same lines, Marc Ongena (University of Agricultural Sciences, Gembloux, Belgium) showed that surfactins (multiple CLP from biocontrol strains of $\mathrm{Ba}$ cillus subtilis) are able to induce plant defense reactions similar to those stimulated by PAMPs. The structure of the lipophilic component of the surfactin seems to influence its activity and correlates well with the surfactin's ability to intercalate into plant membranes.

The effect or production of biosurfactants in different biological systems is dependent on external factors. For example, Dr. Höfte pointed out that pseudomonad CLP would be more effective for biocontrol in sandy soil. As an example of the importance of biosurfactants in tritrophic interactions, Richard Bélanger (Université Laval, Canada) presented evidence that flocculosin (an antifungal cellobiose lipid) is produced by the biocontrol organism Pseudozyma flocculosa under specific conditions linked to the interaction between powdery mildew pathogens and the host plant. The identification of the molecular factors involved in these interactions should help to optimize the use of biosurfactant-producing microbes in the future.

\section{The cell wall.}

The importance of the cell wall and cell-wall modifications in mediating plant-microbe interactions continued to be a ma- 
jor focus. Patrick Schweizer (IPK, Gatersleben, Germany) used high-throughput RNAi-based screening in barley and found a cellulose synthase-like gene, CSLD, that, when mutated, confers hypersusceptibility to Blumeria graminis $\mathrm{f}$. sp. tritici and f. sp. hordei. Bogumil Karas and colleagues (Agriculture and Agri-Food Canada, London, Ontario) found that a CSLD gene in Lotus japonicus, mutation of which causes defects in roothair development, is also required for successful root invasion and nodule formation by Mesorhizobium loti.

An increase in pectin methyl esterification, by downregulation of pectin methylesterase (PME) or overexpression of a PME inhibitor, decreased susceptibility to bacterial and fungal pathogens (Giulia De Lorenzo, University of Rome, Italy). Sheng Yang He (Michigan State University, U.S.A.) elaborated on his work on the type III effector HopM1, showing that it is located in the trans Golgi network, consistent with an involvement in vesicle trafficking and wall reinforcement by papillae in response to pathogen attack. Felice Cervone (University of Rome, Italy) described progress on identification of the receptor for oligogalacturonides (OG), which are one of the oldest known elicitors of defense responses, albeit of plant rather than pathogen origin. OG are released from plant cell walls by the action of pathogen enzymes such as polygalacturonase. By expressing chimeric receptors, Dr. Cervone was able to show that one or more members of the large family of redundant wall-associated protein kinases (WAK) are strong candidates to be one or more of the OG receptors.

Although secretion of a large number of enzymes active on plant cell walls is a dominant feature of most fungi, based on a partial genome survey, the ectomycorrhizal (ECM) fungus Amanita bisporigera is strongly deficient in major classes of these enzymes (Jonathan Walton, Michigan State University, U.S.A.). This had previously been observed by Frances Martin (INRA, Nancy, France) and colleagues in the ECM fungus Laccaria bicolor. This trend appears to hold true for some obligate biotrophic pathogens as well. Laura Baxter (Warwick University, U.K.) and John McDowell (Virginia Bioinformatics Institute, U.S.A.) reported that the genome of Hyaloperonospora arabidopsidis has many fewer genes for cell walldegrading enzymes and other tissue damaging proteins than its relatives in the genus Phytophthora.

\section{Symbiotic interactions.}

The IS-MPMI Congresses are renowned for their cross-disciplinary exchanges. An excellent illustration of this is the area of symbiotic plant-microbe interactions. The research of ISMPMI Congress participants has elucidated close mechanistic connections between bacterial (nitrogen fixation) and fungal (mycorrhizal) symbioses. These connections continued to be a major theme at the Quebec meeting.

Martin Groth and Martin Parniske and colleagues (University of Munich, Germany) described the identification of a signaling network that unites early responses to both types of symbiont. Three putative L. japonicus nucleoporins, Nup133, Nup85, and NENA, are involved in signaling, perhaps by transporting a component essential for calcium spiking in response to Nod factors. Mutants of nena are also impaired in penetration by the mycorrhizal fungus Glomus intraradices. NENA and therefore the nuclear pore complex, apparently has a specific involvement in the regulation of root symbiosis by both bacteria and fungi.

A number of talks unveiled new players in symbiotic interactions. Sharon Long (Stanford University, U.S.A.) presented a plenary talk on new players in symbiotic nitrogen fixation by $S$. meliloti. Flotillins are proteins located in lipid rafts in the root-hair membrane, and they might have a role in endocytosis in infection-thread formation. Other flotillins, like flotillin 2 and 4, are also expressed in nodules. Another exciting new player, $D N F 1$, encodes a signal peptidase required for successful symbiosis.

Bacteroids of $S$. meliloti undergo a remarkable differentiation inside plant roots. Eva Kondorosi (CNRS, Gif-sur-Yvette, France) discussed the role of plant factors of M. truncatula, in particular, nodule-specific antimicrobial peptides (nsAMP or NCR), that are secreted into the symbiosomes and control terminal bacteroid differentiation. These short (60 to 90 amino acids) secreted peptides are found almost exclusively in nodules of galegoid legumes (e.g., genera Medicago, Trifolium, Pisum, and Vicia). The nsAMP family has more than 400 members. Apparently, the target of nsAMP is FtsZ, a protein involved in bacterial and organelle cell division. In the dnfl mutant described by Dr. Long, these proteins remain trapped in the endoplasmic reticulum. In a very elegant approach, they expressed an NCR protein in L. japonicus nodules and showed that this was sufficient to enhance bacterial differentiation.

Jens Stougaard (University of Aarhus, Denmark) combined the L. japonicus snfl gain-of-function allele of CCAMK (calcium and calmodulin-dependent protein kinase) with loss-offunction mutations in common symbiotic genes and, thereby, showed that the function of the latter is dispensable for the infection-thread dependent colonization of root hairs by $M$. loti as long as CCAMK is activated. This significant observation provides increased resolution with respect to plant signaling events that mediate and coordinate root infection by bacteria and nodule primordia organogenesis.

CERBERUS, a novel U-box protein containing WD40 repeats, is required for formation of the infection thread and nodule development in the symbiosis between $L$. japonicus and M. loti (Krzysztof Szczyglowski, Agriculture and AgriFood Canada, London, Ontario). Several of these genes are only involved in the formation of the infection thread without affecting the formation of the nodule meristem.

Maria Harrison (Boyce Thompson Institute, U.S.A.) discussed novel mutants of $M$. truncatula that are affected in mycorrhizal penetration. One particular mutant allows initiation of the formation of the appresorium but not penetration of the plant cell wall. Another mutant is in the STR gene encoding a member of the ABC superfamily of transporters, and a third gene, $M t V P Y$, is a member of the plant-specific vapirin gene family. Interestingly, there are orthologs of this last gene in monocotyledons and dicotyledons but not in Arabidopsis, a nonmycorrhizal plant. $M t V P Y$ is induced in mycorrhizal roots, and knockdown by RNAi impairs root penetration and abolishes arbuscule formation. The gene product contains two protein-protein interaction domains (VAP/MSP and WD40 repeats). Dr. Harrison hypothesizes that this gene may act as a scaffold during fungal penetration of epidermal and cortical cells by binding multiple proteins.

Thomas Ott and Katalin Toth and colleagues (University of Munich, Germany) reported on a member of the remorin scaffold protein family that controls infection by rhizobia. The gene is induced by Nod factors. Remorins had previously been discovered to have a role in movement of Potato virus $X$ in solanaceous plants. Another remorin family member is required for mycorrhizal interactions. Remorin binds the NFR1 and NFR5 Nod receptors in L. japonicus (Benoit Lefèbvre, INRACNRS, Castanet-Tolosan, France).

Anton Wasson and Ulrike Mathesius and colleagues (Australian National University, Canberra) presented an extensive and systematic analysis supporting the key role of auxin transport "at the root of nodule development." Dr. Mathesius described their findings with lateral root development as a reference scenario. Flavonoids and cytokinins play critical roles in nodulation. Malick Mbengue (CNRS, Castanent-Tolosan, 
France) introduced a new $M$. truncatula protein, LUPI, as a putative interactor of the Nod factor receptors LYK3 and DMI2. LUPI is an E3 ligase of the U-box family.

\section{Satellite symposium on powdery mildew genetics.}

More than 70 scientists attended the evening powdery mildew genetics symposium on this important group of fungal plant pathogens organized by Pietro Spanu (Imperial College, London, U.K.) and Ralph Panstruga (Max Planck Institute, Köln, Germany). Major progress on the genome sequencing of Blumeria, Erysiphe, and Golovinomyces was reported. Hans Thordal-Christensen (University of Copenhagen, Denmark) and Lance Cadle-Davidson (USDA, Geneva, NY, U.S.A.) discussed recent advances on mildew avr genes and proteins, and Patrick Schweizer (IPK, Gatersleben, Germany) reported on the identification of basal and nonhost resistance genes in Blumeria-cereal interactions. Yuling Bai (Wageningen University, Netherlands) engineered resistance against tomato pow- dery mildew with a tomato homolog of the barley mlo gene. Roger Wise (Iowa State University, U.S.A.) reported how barley $H v R R P 46$, which encodes an RNAse, induces resistance against Blumeria spp., perhaps by interfering with ribosomal processing. He also discussed a barley protein, BLUFENSIN1 (BLN1), which is highly induced in barley by infection with Blumeria spp. BLN1 is a secreted cysteine-rich protein related to knottins. Ulrich Schaffrath (Aachen University, Germany) used Magnaporthe grisea to investigate powdery mildew biology; members of genus Blumeria induce susceptibility in nonhost plants to $M$. grisea, suggesting that Blumeria spp. secrete suppressors of defense.

The meeting concluded with the inauguration of Felice Cervone (University of Rome, Italy) as the new president of IS-

MPMI. Outgoing president Federico Sánchez (Universidad Nacional Autónoma, Cuernavaca, Mexico) performed the tradition-filled ceremony. Plans for the 15th IS-MPMI Congress in 2011 in Kyoto, Japan, are now underway. 